\title{
REDUCING DOWNLOAD TIME THROUGH MIRROR SERVERS
}

\author{
Renuka Gowardhan ${ }^{1}$, Avinash Dhole ${ }^{2}$, Sanjay Kumar ${ }^{3}$ \\ ${ }^{1}$ Student, ${ }^{2}$ HOD, Department of Computer Science \& Engineering, Raipur Institute of Technology, CG, India \\ ${ }^{3}$ HOD, Department of Computer Science \& Engineering, PRSU, CG, India \\ renuka.gowardhan@gmail.com,avi_dhole33@rediffmail.com
}

\begin{abstract}
Downloading large files on internet is very time consuming and usually slow process. As internet is expanding and the size of data on internet is also become larger the user is in need of the download approach which reduces the download time. Parallelization can be the motivation in the direction to download time reduction. The most reliable protocol which is most widely used is File Transfer Protocol. On internet files are replicated on number of servers. These duplicate servers are called Mirror servers which are designed to provide reliability and increase availability. In traditional FTP the client tries to download a file from the single servers. The selection of server is done on the basis of distance. Generally the server which is geographically closest to the client is selected for file download. Parallel FTP proposes simultaneous downloads of disjoint file portion from multiple servers. Parallel FTP server selects file server for the requesting client on the basis of variety of QoS parameter such as Available Bandwidth and Server Utilization. We perform a simulation based comparative study between Traditional FTP and Parallel FTP. In our approach we also tried to add some more QoS parameter which are Hop Counts and Delay. The file download time is inversely proportional to number of hops and directly proportional to idleness of servers. The simulation is done on Network Simulator -2.
\end{abstract}

Index Terms: Hop Counts, Protocol, Delay, Mirror Server, Replication

\section{INTRODUCTION}

Downloading a large file on internet is a slow and time consuming process. Users require an efficient technique which can reduce the download time. Parallelization can be a major motivation towards Download time reduction. Internet is a wide collection of interconnected computers allowed to use common protocols to server million of users over the world. In a network each channel has certain capacity for transmitting information called bandwidth. During the transmission of data resource availability can change drastically. This can make download process very slow. Delay is the total time required to reach the destination. Hop count is the number of devices through which data or packet is travelled.

Downloading is the process of transferring a file from server to our machine. A Protocol is set of rules which are responsible for end to end transmission. Mirror servers are the exact replicated servers of file servers which are used to provide the reliable access to large downloads. FTP is the most reliable data transfer. In network a file is stored in multiple servers for load distribution.

QoS factors which affect the downloading are Bandwidth, delay, Variation in delay, synchronization between multiple streams, server availability and utilization. Study of traffic parameters have shown that file size distribution is unbounded on size.

Common technique or frame work to reduce download time is parallelization. Initially server selection techniques Involve selecting a server which is geographically closest to client. Various studies concluded that the server which is closest must be highest congestion and causes delay ${ }^{[]}$.In parallelization instead of downloading a file from single server mirror servers are used and disjoint portions of a file can be downloaded simultaneously in parallel.

Parallel FTP scheme contains a PFTP server within each client system. The PFTP file server is capable of communication with file servers located on different places in internet. In Parallel FTP client initially contacts the PFTP server when a particular file is to be downloaded. The PFTP server then selects the suitable mirror servers from which the file can be downloaded in parallel. This information is sent back to the client who then initiates Parallel FTP session. Selection of suitability Mirror servers is done on the basis of QoS parameters.

Our paper is organized as follows Section 2 provide the related work to our approaches. Section 3 provides details on Parallel FTP approach and simulation techniques implementation to perform measures. Next section covers the result of comparative studies and future scope of our approach. 


\section{RELATED WORK}

Initially study has been done and researchers proposed different techniques for selecting mirror server. These techniques are based on single mirror server and client communication. The work similar to our technique is Dynamic Parallel Access Technique. The file is partitioned into small blocks which are downloaded from all mirror servers parallel. The server which is highest throughput sends largest part of file. Dynamic Parallel Access Technique uses the server selection algorithm based on bandwidth prediction performed best. DPAT can be implemented on internet without any modification at mirror servers. DPAT proposes downloading large file by multiple HTTP servers simultaneously.

DPAT proposes downloading large files by connecting to multiple HTTP servers simultaneously. The file is partitioned into small blocks and client requests a block from each server. When the transfer of one block from any mirror server is completed, the client requests the next block from the same server until the complete file is received.

Another limitation of DPAT is that the mirror servers do not have any knowledge about the next block of file they are expected to send until client request for download.

Many Peer- to- Peer application provide simultaneous and partial download. The limitation of Peer-to-Peer applications is application must be installed in all machines which are participated in file sharing. Almost all peer-to-peer approaches allow users to download files directly from the peer who has the copy of desired file. The peer-to-peer approaches tries to facilitate file sharing among all servers without central entity called server. Many peer-to- peer applications provide the advantage of parallel (partial) data transfer. For each data transfer unknown machines or remote machines are allowed and need to be trusted.

Parallel FTP approach works in continuous connection establishment without requiring multiple repeated connections.

\section{METHODOLOGY}

We are presenting an approach to optimize the process of downloading using File Transfer Protocol by selecting multiple servers. The criteria of selecting mirror servers are server availability, Delay, Hop Counts and Bandwidth. Parallelization reduces the burden on any single server path. QoS parameters are used to check the suitability of servers. The portion of file downloaded from each server is distributed on the basis of suitability.

Parallel FTP is implemented on application level. Three main working entities of Parallel FTP are.

\section{PFTP Server}

2. PFTP Client
3. Mirror Servers

For each download client sends a request to PFTP server. When PFTP server receives a request it gather information for each mirror server and executes the suitability algorithm to find out the best suited servers who contain the requested file. Suitability is calculated in terms of Hop counts, resource availability and delay. On the basis of respective suitability the file is distributed.

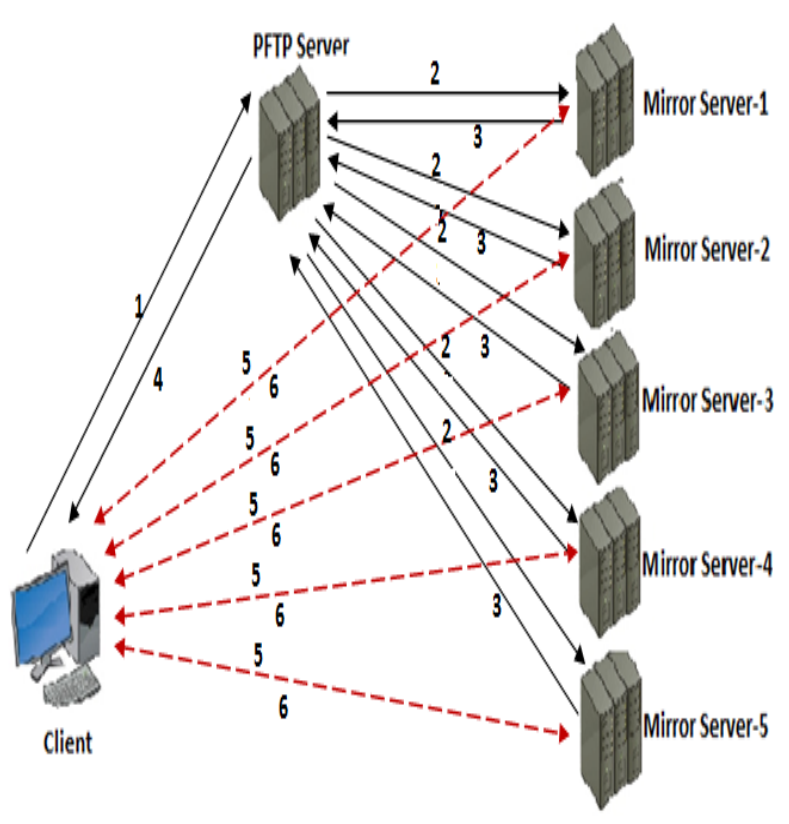

Fig -1: Parallel FTP entities interaction

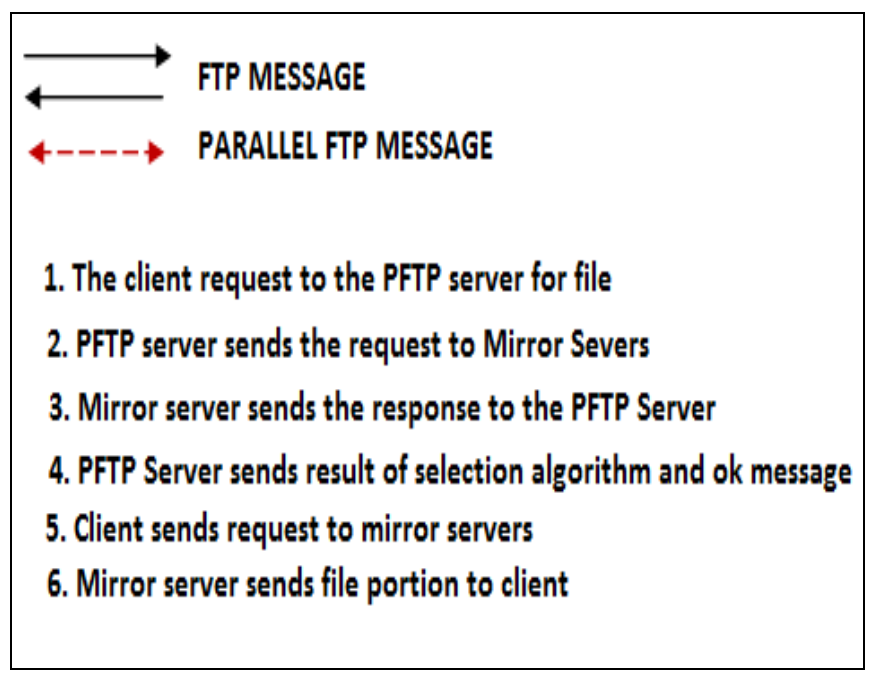

Fig -2 : Parallel FTP entities communication stages

After getting the suitability PFTP server sends this information with an inform message containing suitability and 
client details to all mirror servers. The mirror server then sends a confirm message to PFTP server. PFTP server sends a reply to requesting client with selected mirror server information and suitability. The reply message from PFTP server tells client to what amount of file is downloaded from each mirror server. Client uses this information to reassemble the file after session is ended and download is completed.

After completing the transmission the mirror servers sends complete message to PFTP server, PFTP then deactivate the session. Suitability of mirror servers is calculated recursively for each download request and database is updated accordingly.

\section{Parallel FTP Server:}

It is the central entity of our framework which is places within the system of each client and can be accessed by peers close to it.

\section{Parallel FTP Database:}

The database contains data about network like file server utilization, Hop counts, Delay and list of files on mirror servers. Server utilization can be measured as server's memory and CPU utilization. A special client is placed at mirror servers and sends information about mirror server utilization. File information consist of file replication map of mirror servers that indicates which file is replicated at which server.

\section{Parallel FTP Client:}

The Parallel FTP client is an FTP client with additional capabilities of partial file transfer and flow monitoring. A Parallel FTP client measures the available bandwidth before sending request to retrieve information from PFTP server. PFTP Client sends a request containing a file name and available bandwidth to PFTP server. After getting reply from server the PFTP client establish session with selected mirrors and download disjoint portions of requested file partially. PFTP adjust according to dynamic QoS of network. A client continuously monitors the data transfer rate from all mirror servers. If any mirror servers transfers the data below the expected value the file size is reduced and distributed to other servers.

\section{Ranking and Selection Process:}

Ranking of suitable mirror server is done on the basis of following information such as bandwidth, Hops , Delay, Server availability.

\section{Suitability Algorithm:}

The core of the Parallel FTP is suitability algorithm. After receiving the request from client PFTP server runs suitability algorithm to find the set of suitable servers. The suitability is used to decide the portion of file downloaded from each mirror server.
Suppose the client requests for file F, PFTP server finds the resources required to download the file, FR and the set of mirror servers, $\mathrm{M}$ having file $\mathrm{F}$.

$\mathrm{M} \subset \mathrm{MS}$

Where MS is a set of mirror servers, that are registered with PFTP server. The suitability algorithm finds the suitability Sm for all members of $\mathrm{m}$ on the basis of optimization variables. The suitability of mirror servers are checked for Resource availability AR. The value of Optimization variable must be less than the threshold value.

MFinal $\subset$ MS

MFinal $\subset \mathrm{M}$

Here MFinal is the set of mirror servers to whom PFTP server will send inform message after suitability algorithm finishes. We assumed server availability from 0-1 randomly. These parameters are called optimization parameters. On the basis of these optimization variables suitability of each server is calculated and then individual variables are combined to calculate the overall suitability of server. Let $M$ servers contains the required file. Suitability on the basis of each optimization variable $\mathrm{OV}$ is to be calculated. In our case bandwidth, server availability is Direct Optimization Variables. Hop counts and Delay are Indirect Optimization Variables.

Let Resource availability of $\mathrm{m}$ mirror servers is RAVm then suitability Sk can be calculated as

$\mathrm{Sk}=\frac{\mathrm{RAVm}}{\sum_{\mathrm{i}=1}^{\mathrm{m}} \mathrm{RAVm}}$

$\mathrm{Sk}$ on the basis of bandwidth BWm is calculated as

$\mathrm{Sk}=\frac{\mathrm{BWm}}{\sum_{\mathrm{i}=1}^{\mathrm{m}} \mathrm{BWm}}$

Sk according to Delay Dm is calculated as

$\mathrm{Sk}=\frac{\frac{1}{\mathrm{Dk}}}{\sum_{\mathrm{i}=1}^{\mathrm{m}} \frac{1}{\mathrm{Dk}}}$

Here HCk indicate the hop counts which have inverse effect on download time, so in this case $\mathrm{Sk}$ for $\mathrm{HCk}$ can be calculated as:

$\mathrm{Sk}=\frac{\mathrm{HCk}}{\sum_{\mathrm{i}=1}^{\mathrm{m}} \mathrm{HCk}}$

These suit abilities are added and combines suitability for each mirror server is then calculated. On the basis of this findings mirror servers are ranked and File is partitioned and allocated 
according to ranks. Largest part is allocated to the first ranked computer.

\section{Server Suitability Algorithm:}

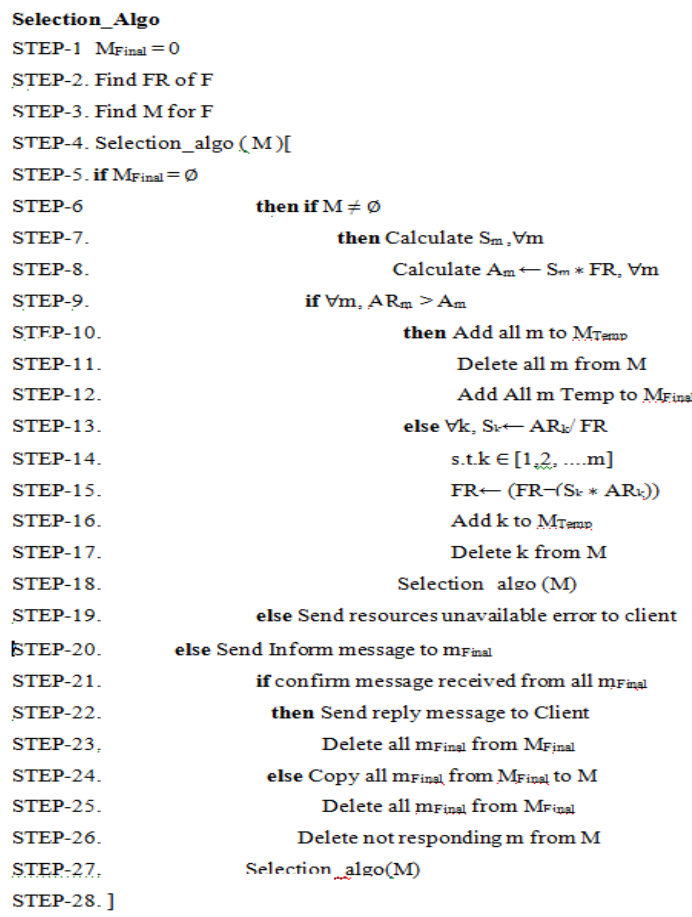

\section{Client Suitability Algorithm:}

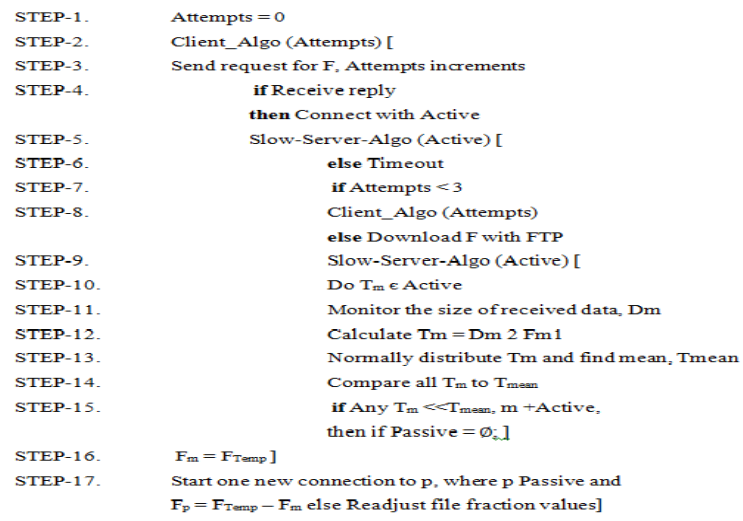

\section{SIMULATION}

Following are the topologies considered for our comparative study:

1. A topology with one client and 10servers, 10 routers and 5 clients which are linked together.

2. The interaction within this topology is dynamic

3. Data Travelling Capacity between client to router $5 \mathrm{mbps}$

4. Data Travelling Capacity between router to router $10 \mathrm{mbps}$
5. Data Travelling Capacity between server to router $5 \mathrm{mbps}$.

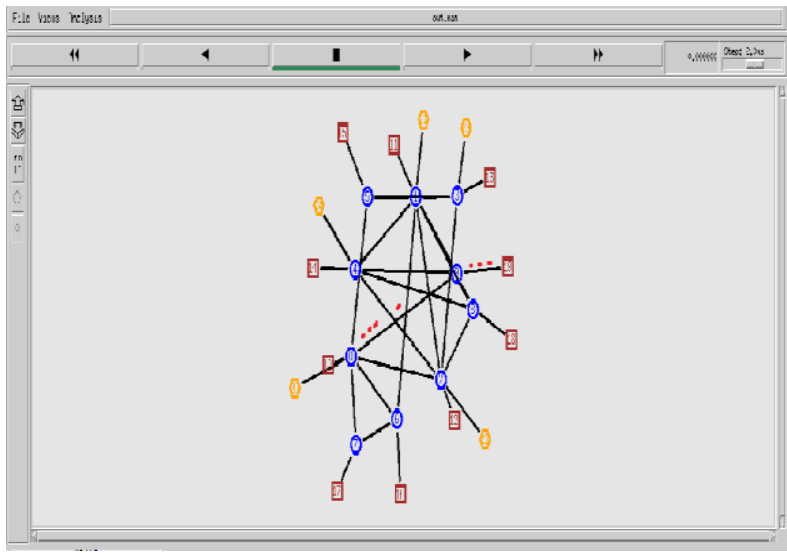

Fig -2: Topology

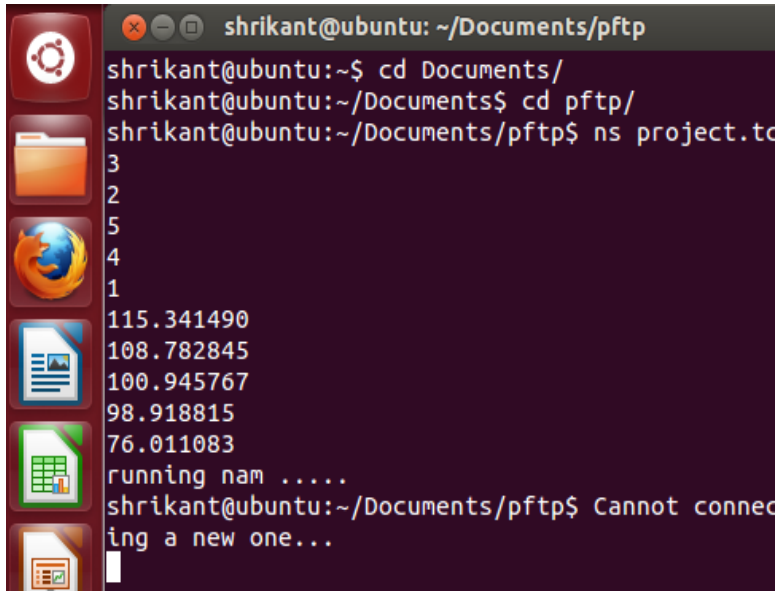

We assumed there are maximum 5 mirror servers containing replicas. We have added the two more QoS parameters to the suitability algorithm which are Hop Counts and Server Availability.

\section{RESULT \& FUTURE SCOPE}

Use of multiple mirror servers to download a large file is the best suited download to give fastest download. In this paper we have simulated and compared the Traditional FTP and Our Parallel FTP variation.

Table -1: Download time

\begin{tabular}{|l|c|c|c|c|}
\hline Techniques & $100 \mathrm{~kb}$ & $200 \mathrm{~kb}$ & $250 \mathrm{~kb}$ & $300 \mathrm{~kb}$ \\
\hline FTP & 0.7610 & 1.3390 & 1.6939 & 1.9200 \\
\hline PFTP & 0.3192 & 0.3990 & 0.4889 & 0.5040 \\
\hline
\end{tabular}


A server which runs the suitability algorithm also maintained a database which contains number of hops between mirror servers to client, idleness of each mirror server and percentage of file to be downloaded from each server.

Parallel FTP is fair as the selection of mirror servers is based on the available bandwidth between client and internet gateway. PFTP is byte oriented approach and less data loss is done if server fails only the penalty is client need to establish connection again which require extra time.

The ability of our protocol is it can dynamically update the division of file during transfer. Further studies can be done for the dynamic selection of different file servers for each request. More studies on routing algorithms should be done. The routing algorithm adopted for transmission should find the path which may not be shortest but it should be best suited path.

\section{ACKNOLEDGEMENTS}

I want to thank Dr. Sanjay Kumar for his valuable feedback and guidance and Mr. Avinash Dhole for guiding me and supporting me in my research work.

\section{REFERENCES:}

[1]. J. Guyton and M. Schwartz, "Locating Nearby Copies of Replicated Internet Servers", In Proceedings of SIGCOMM 1995, pages 288-298.

[2]. Z. Fu and N. Venkatasubramanian, "Directory Based Composite Routing and Scheduling Policies for Dynamic Multimedia Environment", In Proceedings of IEEE International Parallel and Distributed Processing Symposium 2001 (IPDPS 2001)

[3]. P. Rodriguez and E.Biersack, "Dynamic Parallel Access to Replicated Content in the Internet, "IEEE/ACM Trans. Networking, vol. 10, no. 4, pp. 455-465, Aug. 2002.

[4]. S. Sohail, S. Jha, and H. Elgindy, "Parallelized File Transfer Protocol,'Proc. IEEE Local Computer Networks Workshop High-Speed Local Networks,pp. 624-631, 2003.

[5] S. Sohail and S. Jha, "QoS Driven Parallelized FileTransfer-Protocol,'Proc. Australian Telecomm., Networks, and Application Conf.,2004.

[6] Renuka Gowardhan, Mr. Avinash Dhole and Dr. Sanjay Kumar "a Variation in Parallel File Transfer Protocol", Shastrarth-2013, An International Multidisciplinary Conference organized by Rungta Group of Colleges, Bhilai Chhattisgarh, India.

[7] www.wikipedia.com 